\title{
A Contemporary Assessment of Mechanical Complication Rates and Trainee Perceptions of Central Venous Catheter Insertion
}

\author{
Lauren Heidemann, $\mathrm{MD}^{1,2}$, Niket Nathani, $\mathrm{MD}^{3}$, Rommel Sagana, $\mathrm{MD}^{3}$, Vineet Chopra, MD, MSc ${ }^{1,2}$, Michael Heung, MD, MS ${ }^{4 *}$
}

${ }^{1}$ Department of Internal Medicine, University of Michigan, Ann Arbor, Michigan; ${ }^{2}$ A A Ann Arbor Healthcare System, Ann Arbor, Michigan; ${ }^{3}$ Department of Internal Medicine, Division of Pulmonary and Critical Care Medicine, University of Michigan, Ann Arbor, Michigan; ${ }^{4}$ Department of Internal Medicine, Division of Nephrology, University of Michigan, Ann Arbor, Michigan.

BACKGROUND: Limited data exist regarding rates of mechanical complications of ultrasound-guided, nontunneled central venous catheters (CVC). Similarly, trainee perceptions surrounding CVC complications are largely unknown.

OBJECTIVES: To evaluate contemporary CVC mechanical complication rates, associated risk factors, and trainee perspectives.

DESIGN: A single-center retrospective review of CVC procedures between June 1, 2014, and May 1, 2015. Electronic survey distributed to internal medicine trainees.

SETTING: Intensive care units and the emergency department at an academic hospital.

MEASUREMENTS: Electronic health records of patients with CVC procedures were reviewed for complications. Demographic and procedural characteristics were compared for complicated vs uncomplicated procedures. Student $t$ tests and chi-square tests were used to compare continuous and categorical variables, respectively.
RESULTS: Of the 730 reviewed records, 14 serious mechanical complications occurred due to pneumothorax $(n=5)$, bleeding $(n=3)$, vascular injury $(n=3)$, stroke $(n=1)$, and death $(n=2)$. Risk factors for complicated vs uncomplicated CVC placement included subclavian location $(21.4 \%$ vs $7.8 \%$, $P=0.001)$, number of attempts (2.2 vs $1.5, P=0.02)$, unsuccessful CVC (21.4\% vs. $4.3 \%, P=0.001)$, attending supervision (61.5\% vs $34.7 \%, P=0.04)$, low body mass index (mean $25.7 \mathrm{~kg} /$ $\mathrm{m}^{2}$ vs $\left.31.5 \mathrm{~kg} / \mathrm{m}^{2}, P=0.001\right)$, anticoagulation (28.6\% vs $20.6 \%$, $P=0.048)$, and ventilation (78.5\% vs $66.5 \%, P=0.001)$. Survey data suggested deficiencies in managing unsuccessful CVC procedures; specifically, only $35 \%(N=21 / 60)$ of trainees regularly perform chest $\mathrm{x}$-rays after failed CVC attempt.

CONCLUSIONS: We observed a $1.9 \%$ rate of mechanical complications associated with CVC placement. Our study confirms historical data that unsuccessful CVC attempts are an important risk factor for complications. Education regarding unsuccessful CVC placement may improve patient safety. Journal of Hospital Medicine 2017;12:646-651. (C) 2017 Society of Hospital Medicine
Central venous catheter (CVC) placement is commonly performed in emergency and critical care settings for parenteral access, central monitoring, and hemodialysis. Although potentially lifesaving CVC insertion is associated with immediate risks including injury to nerves, vessels, and lungs. ${ }^{1-3}$ These "insertion-related complications" are of particular interest for several reasons. First, the frequency of such complications varies widely, with published rates between $1.4 \%$ and $33.2 \% .^{2-7}$ Reasons for such variation include differences in study definitions of complications (eg, pneumothorax and tip position), ${ }^{2,5}$ setting of CVC placement (eg, intensive care unit [ICU] vs emergency room), timing of placement (eg, elective vs emergent), differences in technique, and type of operator (eg, experienced vs learner). Thus, the precise incidence of such events in modern-day training settings with use of ultra-

\footnotetext{
*Address for correspondence and reprint requests: Michael Heung, MD, MS, 1500 E. Medical Center Drive, SC 5364, Ann Arbor, MI 48109-5364; Telephone: 734-936-6489; Fax: 734-936-9621; E-mail: mheung@umich.edu

Additional Supporting Information may be found in the online version of this article.

Received: December 12, 2016; Revised: January 25, 2017;

Accepted: February 5, 2017
}

2017 Society of Hospital Medicine DOI 10.12788/jhm.2784 sound guidance remains uncertain. Second, mechanical complications might be preventable with adequate training and supervision. Indeed, studies using simulation-based mastery techniques have demonstrated a reduction in rates of complications following intensive training. ${ }^{8}$ Finally, understanding risk factors associated with insertion complications might inform preventative strategies and improve patient safety. ${ }^{9-11}$

Few studies to date have examined trainees' perceptions on CVC training, experience, supervision, and ability to recognize and prevent mechanical complications. While research investigating effects of simulation training has accumulated, most focus on successful completion of the procedure or individual procedural steps with little emphasis on operator perceptions. ${ }^{12-14}$ In addition, while multiple studies have shown that unsuccessful line attempts are a risk factor for CVC complications, , $^{3,7,15}$ there is very little known about trainee behavior and perceptions regarding unsuccessful line placement. CVC simulation trainings often assume successful completion of the procedure and do not address the crucial postprocedure steps that should be undertaken if a procedure is unsuccessful. For these reasons, we developed a survey to specifically examine trainee experience with CVC placement, supervision, postprocedural behavior, and attitudes regarding unsuccessful line placement. 
Therefore, we designed a study with 2 specific goals: The first is to perform a contemporary analysis of CVC mechanical complication rate at an academic teaching institution and identify potential risk factors associated with these complications. Second, we sought to determine trainee perceptions regarding CVC complication experience, prevention, procedural supervision, and perceptions surrounding unsuccessful line placement.

\section{METHODS}

\section{Design and Setting}

We conducted a single-center retrospective review of nontunneled acute CVC procedures between June 1, 2014, and May 1, 2015, at the University of Michigan Health System (UMHS). UMHS is a tertiary care referral center with over 900 inpatient beds, including 99 ICU beds.

All residents in internal medicine, surgery, anesthesia, and emergency medicine receive mandatory education in CVC placement that includes an online training module and simulation-based training with competency assessment. Use of real-time ultrasound guidance is considered the standard of care for CVC placement.

\section{Data Collection}

Inpatient procedure notes were electronically searched for terms indicating CVC placement. This was performed by using our hospital's Data Office for Clinical and Translational Research using the Electronic Medical Record Search Engine tool. Please see the supplemental materials for the full list of search terms. We electronically extracted data, including date of procedure, gender, and most recent body mass index (BMI), within 1 year prior to note. Acute Physiology and Chronic Health Evaluation III (APACHE III) data are tracked for all patients on admission to ICU; this was collected when available. Charts were then manually reviewed to collect additional data, including international normalized ratio (INR), platelet count, lactate level on the day of CVC placement, anticoagulant use (actively prescribed coumadin, therapeutic enoxaparin, therapeutic unfractionated heparin, or direct oral anticoagulant), ventilator or noninvasive positive pressure ventilation (NIPPV) at time of CVC placement, and vasopressor requirement within 24 hours of $\mathrm{CVC}$ placement. The procedure note was reviewed to gather information about site of CVC placement, size and type of catheter, number of attempts, procedural success, training level of the operator, and attending presence. Small bore CVCs were defined as 7 French $(\mathrm{Fr})$ or lower. Large bore CVCs were defined as $>7 \mathrm{Fr}$; this includes dialysis catheters, Cordis catheters (Cordis, Fremont, CA), and cooling catheters. The times of the procedure note and postprocedure chest $\mathrm{x}$-ray (CXR) were recorded, including whether the CVC was placed on a weekend (Friday 7 PM to Sunday at midnight) or weekday.

\section{Primary Outcome}

The primary outcome was the rate of severe mechanical complications related to CVC placement. Similar to prior studies, ${ }^{2}$ we defined severe mechanical complications as arterial placement of dilator or catheter, hemothorax, pneumothorax, cerebral ischemia, patient death (related to procedure), significant hematoma, or vascular injury (defined as complication requiring expert consultation or blood product transfusion). We did not require a lower limit on blood transfusion. We considered pneumothorax a complication regardless of whether chest tube intervention was performed, as pneumothorax subjects the patient to additional tests (eg, serial CXRs) and sometimes symptoms (shortness of breath, pain, anxiety) regardless of whether or not a chest tube was required. Complications were confirmed by a direct review of procedure notes, progress notes, discharge summaries, and imaging studies.

\section{Trainee Survey}

A survey was electronically disseminated to all internal medicine and medicine-pediatric residents to inquire about CVC experiences, including time spent in the medical ICU, number of CVCs performed, postprocedure behavior for both failed and successful CVCs, and supervision experience and attitudes. Please see supplemental materials for full survey contents.

\section{Statistical Methods}

Descriptive statistics (percentage) were used to summarize data. Continuous and categorical variables were compared using Student $t$ tests and chi-square tests, respectively. All analyses were performed using SAS 9.3 (SAS Institute, Cary, NC).

\section{Ethical and Regulatory Oversight}

The study was deemed exempt by the University of Michigan Institutional Review Board (HUM00100549) as data collection was part of a quality improvement effort.

\section{RESULTS}

\section{Demographics and Characteristics of Device Insertion}

Between June 1, 2014, and May 1, 2015, 730 CVC procedure notes were reviewed (Table 1 ). The mean age of the study population was 58.9 years, and $41.6 \%(n=304)$ were female. BMI data were available in 400 patients without complications and 5 patients with complications; the average BMI was 31.5 $\mathrm{kg} / \mathrm{m}^{2}$. The APACHE III score was available for 442 patients without complications and 10 patients with complications; the average score was 86 (range 19-200). Most of the CVCs placed $(n=504,69 \%)$ were small bore $(<7 \mathrm{Fr})$. The majority of catheters were placed in the internal jugular (IJ) position $(n=525$, $71.9 \%)$, followed by femoral $(\mathrm{n}=144,19.7 \%)$, subclavian $(\mathrm{N}$ $=57,7.8 \%)$, and undocumented $(n=4,0.6 \%)$. Ninety-six percent $(n=699)$ of CVCs were successfully placed. Seventy-six percent $(n=558)$ of procedure notes included documentation of the number of CVC attempts; of these, $85 \%$ documented 2 or fewer attempts. The majority of CVCs were placed by residents $(\mathrm{n}=537,73.9 \%)$, followed by fellows $(\mathrm{N}=127,17.5 \%)$ and attendings $(\mathrm{n}=27,3.7 \%)$. Attending supervision for all or key portions of CVC placement occurred 34.7\% $(n=244)$ of 
TABLE 1. Demographic and Procedural Characteristics of Complicated vs Uncomplicated CVC Placement.

\begin{tabular}{|c|c|c|c|c|}
\hline Characteristics & Overall Study Cohort & $\begin{array}{c}\text { Mechanical CVC } \\
\text { Complication }\end{array}$ & $\begin{array}{c}\text { No Mechanical CVC } \\
\text { Complication }\end{array}$ & $P$ Value \\
\hline Total number & 730 & 14 & 716 & \\
\hline Gender, n (\% female) & $304(41.6 \%)$ & $8(57.1 \%)$ & $296(41.3 \%)$ & .235 \\
\hline Service, \#CVC (\%) & & & & .494 \\
\hline Medicine & $465(63.7 \%)$ & $10(71.4 \%)$ & $455(63.6 \%)$ & \\
\hline Anesthesia & $18(2.5 \%)$ & $0(0 \%)$ & $18(2.5 \%)$ & \\
\hline BMI, average, $\mathrm{kg} / \mathrm{m}^{2}$ (SD) & $31.5(14.9)$ & $25.7(2.1)$ & $31.0(12.7)$ & .001 \\
\hline CVC Typea, n (\%) & & & & .821 \\
\hline Small bore & $504(69.0 \%)$ & 10 (71.4\%) & $494(69.0 \%)$ & \\
\hline Large Bore & $193(26.4 \%)$ & $3(21.4 \%)$ & $190(26.5 \%)$ & \\
\hline Subclavian & $57(7.8 \%)$ & $3(21.4 \%)$ & $54(7.5 \%)$ & \\
\hline Unknown & $4(0.6 \%)$ & $1(7.1 \%)$ & $3(0.4 \%)$ & \\
\hline CVC side, n (\%) & & & & .146 \\
\hline Right & $381(72.7 \%)$ & $4(50 \%)$ & 377 (73.1\%) & \\
\hline Left & $143(27.3 \%)$ & $4(50 \%)$ & 139 (26.9\%) & \\
\hline \# of Attempts, mean (SD) & $1.5(0.9)$ & $2.2(1.2)$ & $1.5(0.9)$ & .020 \\
\hline Successful CVC, n (\%) & $699(95.7 \%)$ & $11(78.6 \%)$ & 688 (96.1\%) & .001 \\
\hline Year of training, $n$ (\%) & & & & .658 \\
\hline First year resident & 165 (22.7\%) & $4(28.6 \%)$ & $161(22.6 \%)$ & \\
\hline All other residents & $372(51.2 \%)$ & $5(35.7 \%)$ & 367 (51.5\%) & \\
\hline First year fellow & 75 (10.3\%) & $2(14.3 \%)$ & $73(10.2 \%)$ & \\
\hline First 6 months of year & 426 (58.3\%) & 10 (71.4\%) & 416 (58.1\%) & \\
\hline Last 5 months of year & $304(41.6 \%)$ & $4(28.6 \%)$ & 300 (41.9\%) & \\
\hline Time of day, $n(\%)$ & & & & .968 \\
\hline 8AM-8PM & $421(57.7 \%)$ & $8(57.1 \%)$ & $413(57.7 \%)$ & \\
\hline 8PM-8AM & 309 (42.3\%) & $6(42.9 \%)$ & $303(42.3 \%)$ & \\
\hline Weekend vs weekday, n (\%) & & & & .125 \\
\hline Weekend & 322 (44.1\%) & $9(64.3 \%)$ & 313 (43.8\%) & \\
\hline Weekday & $408(55.9 \%)$ & $5(35.7 \%)$ & $403(56.2 \%)$ & \\
\hline APACHE III score, mean (range) on day of admission & $86(19-200)$ & $96(57-157)$ & $86(19-200)$ & .397 \\
\hline
\end{tabular}

aSmall bore CVCs include single lumen, double lumen, and triple lumen catheters. Large bore CVCs include dialysis catheters, Cordis, and cooling catheters.

bJ line placement only.

NOTE: Abbreviations: APACHE III, Acute Physiology and Chronic Health Evaluation III; BMI, Body Mass Index; CVC, central venous catheter; IJ, internal jugular; SD, standard deviation.

the time overall and was lower for internal medicine trainees $(\mathrm{n}=98 / 463,21.2 \%)$ compared with surgical trainees $(\mathrm{n}=$ $73 / 127,57.4 \%)$ or emergency medicine trainees $(n=62 / 96$, 64.6\%; P $<0.001$ ). All successful IJ and subclavian CVCs except for 2 insertions $(0.3 \%)$ had a postprocedure CXR. A minority of notes documented pressure transduction $(4.5 \%)$ or blood gas analysis $(0.2 \%)$ to confirm venous placement.
Lab data, information on utilization of anticoagulation, vasopressors, ventilation, and information on the use of transduction and blood gas data were collected for the first 410 uncomplicated patients and all patients that experienced complications (Table 2). The mean INR was 1.5 (range 0.9. 9.7), mean platelets $180 \mathrm{~K} / \mathrm{uL}$ (range $9-816 \mathrm{~K} / \mathrm{uL}$ ), and mean lactate $2.9 \mathrm{mmol} / \mathrm{L}$ (range $0.4-16 \mathrm{mmol} / \mathrm{L}$ ). Twenty-one per- 
TABLE 2. Labs and Clinical Status of Complicated vs Uncomplicated CVC Placement ${ }^{a}$

\begin{tabular}{|c|c|c|c|c|}
\hline & \multicolumn{3}{|c|}{ Mechanical CVC } & \multirow[b]{2}{*}{$P$ Value } \\
\hline & Overall Study Cohort & Complication & No CVC Complication & \\
\hline \multicolumn{5}{|c|}{ Systemic anticoagulation ${ }^{\mathrm{b}}, \mathrm{n}(\%)$} \\
\hline None & 331 (79.4\%) & $10(71.4 \%)$ & $321(79.6 \%)$ & .048 \\
\hline \multicolumn{5}{|l|}{ Labs, average (SD) } \\
\hline INR & $1.5(0.9)$ & $1.7(0.9)$ & $1.5(0.9)$ & .538 \\
\hline Vasopressor use, n (\%) & $251(59.2 \%)$ & $10(71.4 \%)$ & 241 (58.8\%) & .344 \\
\hline \multicolumn{5}{|l|}{ Ventilator use, n (\%) } \\
\hline Intubated & $265(62.7 \%)$ & $10(71.4 \%)$ & $255(62.2 \%)$ & .001 \\
\hline NIPPV & $16(3.8 \%)$ & $1(7.1 \%)$ & $15(3.7 \%)$ & \\
\hline None & $140(33.1 \%)$ & $2(14.3 \%)$ & $138(33.5 \%)$ & \\
\hline Unknown & $2(0.5 \%)$ & $1(7.1 \%)$ & $1(0.2 \%)$ & \\
\hline
\end{tabular}

alnformation was gathered for the first 410 uncomplicated procedures (where data were available) and for all procedures associated with complications.

${ }^{\mathrm{D} A n t i c o a g u l a t i o n ~ i n c l u d e s ~ t h e r a p e u t i c ~ h e p a r i n, ~ w a r f a r i n, ~ o r ~ d i r e c t ~ o r a l ~ a n t i c o a g u l a n t . ~}$

NOTE: Abbreviations: CVC, central venous catheter; INR, international normalized tatio; NIPPV, noninvasive positive pressure ventilation; SD, standard deviation.

cent $(\mathrm{n}=86)$ of patients were systemically anticoagulated at the time of CVC placement, $59 \%(\mathrm{n}=251)$ received vasopressors within 24 hours of CVC placement, and 63\% ( $\mathrm{n}=$ 265) were mechanically ventilated (Table 2 ).

\section{Mechanical Complications}

The mechanical complications identified included pneumothorax $(n=5)$, bleeding requiring transfusion $(n=3)$, vascular injury requiring expert consultation or intervention $(n=3)$, stroke $(\mathrm{n}=1)$, and death $(\mathrm{n}=2)$. Vascular injuries included 1 neck hematoma with superinfection requiring antibiotics, 1 neck hematoma requiring otolaryngology and vascular surgery consultation, and 1 venous dissection of IJ vein requiring vascular surgery consultation. None of these cases required operative intervention. The stroke was caused by inadvertent CVC placement into the carotid artery. One patient experienced tension pneumothorax and died due to this complication; this death occurred after 3 failed left subclavian CVC attempts and an ultimately successful CVC placement into left IJ vein. Another death occurred immediately following unsuccessful Cordis placement. As no autopsy was performed, it is impossible to know if the cause of death was the line placement. However, it would be prudent to consider this as a CVC complication given the temporal relationship to line placement. Thus, the total number of patients who experienced severe mechanical complications was 14 out of 730 (1.92\%).

\section{Risk Factors for Mechanical Complications}

Certain patient factors were more commonly associated with complications. For example, BMI was significantly lower in the group that experienced complications vs those that did not $\left(25.7 \mathrm{vs} 31.0 \mathrm{~kg} / \mathrm{m}^{2}, P=0.001\right)$. No other associations between demographic factors, including age (61.4 years vs 58.9 years, $P=0.57)$ or sex $(57.1 \%$ male vs $41.3 \%$ female, $P=$ 0.24), or admission APACHE III score ( 96 vs $86, P=0.397$ ) were noted. The mean INR, platelets, and lactate did not dif- fer between the 2 groups. There was no difference between the use of vasopressors. Ventilator use (including endotracheal tube or NIPPV) was found to be significantly higher in the group that experienced mechanical complications $(78.5 \%$ vs $65.9 \%, P=0.001)$. Anticoagulation use was also associated with mechanical complications $(28.6 \%$ vs $20.6 \%, P=$ $0.05) ; 3$ patients on anticoagulation experienced significant hematomas. Mechanical complications were more common with subclavian location $(21.4 \%$ vs $7.8 \%, P=0.001)$; in all 3 cases involving subclavian CVC placement, the complication experienced was pneumothorax. The number of attempts significantly differed between the 2 groups, with an average of 1.5 attempts in the group without complications and 2.2 attempts in the group that experienced complications $(P=$ 0.02). Additionally, rates of successful placement were lower among patients who experienced complications ( $78.6 \%$ vs 95.7\%, $P=0.001$ ).

With respect to operator characteristics, no significant difference between the levels of training was noted among those who experienced complications vs those who did not. Attending supervision was more frequent for the group that experienced complications $(61.5 \%$ vs $34.2 \%, P=0.04)$. There was no significant difference in complication rate according to the first vs the second half of the academic year $(0.4 \%$ vs $0.3 \%$ per month, $P=0.30)$ or CVC placement during the day vs night $(1.9 \%$ vs $2.0 \%, P=0.97)$. A trend toward more complications in CVCs placed over the weekend compared to a weekday was observed ( $2.80 \%$ vs $1.23 \%$, $P=0.125$ ).

\section{Unsuccessful CVCs}

There were 30 documented unsuccessful CVC procedures, representing $4.1 \%$ of all procedures. Of these, 3 procedures had complications; these included 2 pneumothoraxes (1 leading to death) and 1 unexplained death. Twenty-four of the unsuccessful CVC attempts were in either the subclavi- 
an or IJ location; of these, 5 (21\%) did not have a postprocedure CXR obtained.

\section{Survey Results}

The survey was completed by 103 out of 166 internal medicine residents (62\% response rate). Of these, $55 \%(n=57)$ reported having performed 5 or more CVCs, and 14\% ( $\mathrm{n}=$ 14) had performed more than 15 CVCs.

All respondents who had performed at least $1 \mathrm{CVC}(\mathrm{n}=$ 80) were asked about their perceptions regarding attending supervision. Eighty-one percent $(n=65 / 80)$ responded that they have never been directly supervised by an attending during CVC placement, while $16 \%(n=13 / 80)$ reported being supervised less than $25 \%$ of the time. Most $(n=53 / 75$, $71 \%)$ did not feel that attending supervision affected their performance, while $21 \%(\mathrm{n}=16 / 75)$ felt it affected performance negatively, and only $8 \%(n=6 / 75)$ stated it affected performance positively. Nineteen percent $(n=15 / 80)$ indicated that they prefer more supervision by attendings, while $35 \%(\mathrm{n}=28 / 80)$ did not wish for more attending supervision, and $46 \%(\mathrm{n}=37 / 80)$ were indifferent.

All respondents who had performed at least $1 \mathrm{CVC}$ were asked about postprocedure protocols. The vast majority (n $=77 / 80,95 \%$ ) reported documenting a postprocedure note more than $75 \%$ of the time after a successful procedure; in contrast, only $38 \%(n=30 / 80)$ of those who had failed a CVC placement reported documenting a procedure note more than $75 \%$ of the time (Figure 1$)$. Only $35 \%(n=$ $21 / 60$ ) of respondents reported routinely (100\% of the time) ordering a CXR after a failed chest CVC attempt (Figure $2)$, and $47 \%(n=28 / 60)$ only ordered a CXR if they were concerned there was a complication. Most $(69 \%, n=55 / 80)$ felt it was important to order a CXR after a failed chest CVC placement, while $6 \%(n=5 / 80)$ did not feel it was important, and 25\% ( $\mathrm{n}=20 / 80)$ did not know or were indifferent.

\section{DISCUSSION}

We performed a contemporary analysis of CVC placement at an academic tertiary care center and observed a rate of severe mechanical complications of $1.9 \%$. This rate is within previously described acceptable thresholds. ${ }^{16}$ Our study adds to the literature by identifying several important risk factors for development of mechanical complications. We confirm many risk factors that have been noted historically, such as subclavian line location, ${ }^{2,3}$ attending supervision, ${ }^{3}$ low BMI, ${ }^{4}$ number of CVC attempts, and unsuccessful CVC placement. ${ }^{3,4,7,15}$ We identified several unique risk factors, including systemic anticoagulation as well as ventilator use. Lastly, we identified unexpected deficits in trainee knowledge surrounding management of failed CVCs and negative attitudes regarding attending supervision.

Most existing literature evaluated risk factors for CVC complication prior to routine ultrasound use $;^{3-5,7,15}$ surprisingly, it appears that severe mechanical complications do not differ dramatically in the real-time ultrasound era. Eisen et al. ${ }^{3}$ prospectively studied CVC placement at an academic

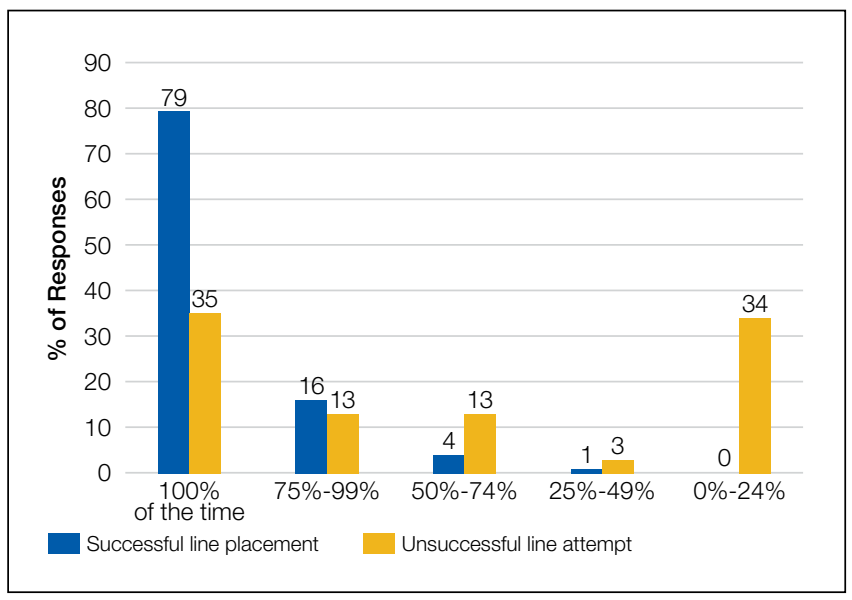

FIG 1. Trainee documentation after successful and unsuccessful CVC attempts. NOTE: Abbreviation: CVC, central venous catheter.

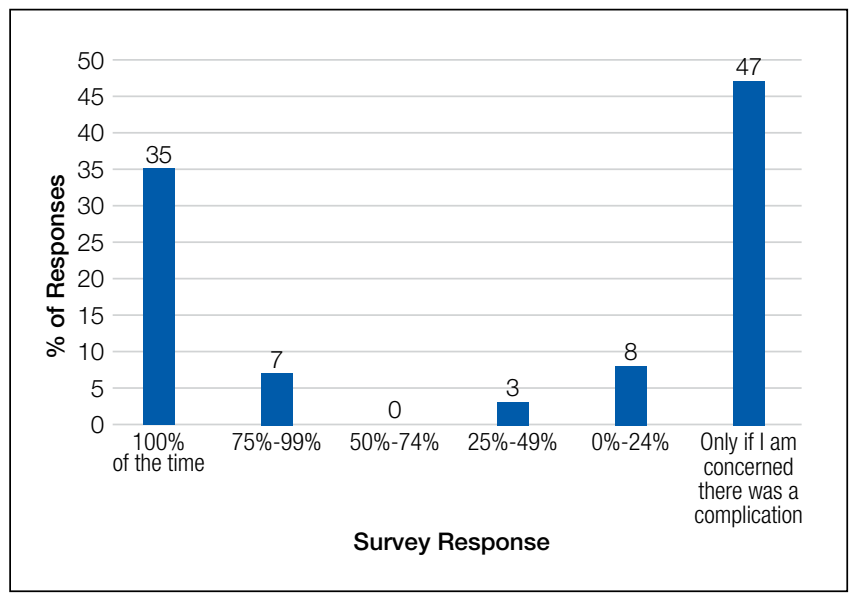

FIG 2. Trainee reported postprocedure CXR ordering after unsuccessful CVC attempt.

NOTE: Abbreviation: CVC, central venous catheter; CXR, chest x-ray.

medical center and found a severe mechanical complication rate (as defined in our paper) of $1.9 \%$ due to pneumothorax $(1.3 \%)$, hemothorax $(0.3 \%)$, and death $(0.3 \%)$. We would expect the number of complications to decrease in the postultrasound era, and indeed it appears that pneumothoraces have decreased likely due to ultrasound guidance and decrease in subclavian location. However, in contrast, rates of significant hematomas and bleeding are higher in our study. Although we are unable to state why this may be the case, increasing use of anticoagulation in the general population might explain this finding. ${ }^{17}$ For instance, of the 6 patients who experienced hematomas or vascular injuries in our study, 3 were on anticoagulation at the time of CVC placement.

Interestingly, time of academic year of CVC placement and level of training were not correlated with an increased risk of complications, nor was time of day of CVC placement. In contrast, Merrer et al. showed that CVC insertion during nighttime was significantly associated with increased mechanical complications (odds ratio 2.06, 95\% confi- 
dence interval, 1.04-4.08;,P $=0.03) . .^{5}$ This difference may be attributable to the fact that most of our ICUs now have a night float system rather than a more traditional 24-hour call model; therefore, trainees are less likely to be sleep deprived during CVC placement at night.

Severity of illness did not appear to significantly affect mechanical complication rates based on similar APACHE scores between the 2 groups. In addition, other indicators of illness severity (vasopressor use or lactate level) did not suggest that sicker patients may be more likely to experience mechanical complications than others. One could conjecture that perhaps sicker patients were more likely to have lines placed by more experienced trainees, although the present study design does not allow us to answer this question. Interestingly, ventilator use was associated with higher rates of complications. We cannot say definitively why this was the case; however, 1 contributing factor may be the physical constraints of placing the CVC around ventilator tubing.

Several unexpected findings surrounding attending supervision were noted: first, attending supervision appears to be significantly associated with increased complication rate, and second, trainees have negative perceptions regarding attending supervision. Eisen et al. showed a similar association between attending supervision and complication rate. ${ }^{3}$ It is possible that the increased complication rate is because sicker patients are more likely to have procedural supervision by attendings, attending physicians may be called to supervise when a CVC placement is not going as planned, or attendings may supervise more inexperienced operators. Reasons behind negative trainee attitudes surrounding supervision are unclear and literature on this topic is limited. This is an area that warrants further exploration in future studies.

Another unexpected finding is trainee practices regarding unsuccessful CVC placement; most trainees do not document failed procedures or order follow-up CXRs after unsuccessful CVC attempts. Given the higher risk of complications after unsuccessful CVCs, it is paramount that all physicians are trained to order postprocedure CXR to rule out pneumothorax or hemothorax. Furthermore, documentation of failed procedures is important for medical accuracy, transparency, and also hospital billing. It is unknown if these practices surrounding unsuccessful CVCs are institution-specific or more widespread. As far as we know, this is the first time that trainee practices regarding failed CVC placement have been published. Interestingly, while many current guidelines call attention to prevention, recognition, and management of central line-associated mechanical complications, specific recommendations about postprocedure behavior after failed CVC placement are not published. ${ }^{9-11}$ We feel it is critical that institutions reflect on their own practices, especially given that unsuccessful CVCs are shown to be correlated with a significant increase in complication rate. At our own institution, we have initiated an educational component of central line training for medicine trainees specifically addressing failed central line attempts.

This study has several limitations, including a retrospec- tive study design at a single institution. There was a low overall number of complications, which reduced our ability to detect risk factors for complications and did not allow us to perform multivariable adjustment. Other limitations are that only documented CVC attempts were recorded and only those that met our search criteria. Lastly, not all notes contain information such as the number of attempts or peer supervision. Furthermore, the definition of CVC "attempt" is left to the operator's discretion.

In conclusion, we observed a modern CVC mechanical complication rate of $1.9 \%$. While the complication rate is similar to previous studies, there appear to be lower rates of pneumothorax and higher rates of bleeding complications. We also identified a deficit in trainee education regarding unsuccessful CVC placement; this is a novel finding and requires further investigation at other centers.

Disclosure: The authors have no conflicts of interest to report.

\section{References}

1. McGee DC, Gould MK. Preventing complications of central venous catheterization. N Engl J Med. 2003;348(12):1123-1133

2. Parienti JJ, Mongardon N, Mégarbane B, et al. Intravascular complications of central venous catheterization by insertion site. N Engl J Med. 2015;373(13):1220-1229.

3. Eisen LA, Narasimhan M, Berger JS, Mayo PH, Rosen MJ, Schneider RF. Mechanical complications of central venous catheters. J Intensive Care Med. 2006;21(1):40-46.

4. Mansfield PF, Hohn DC, Fornage BD, Gregurich MA, Ota DM. Complications and failures of subclavian-vein catheterization. N Engl J Med. 1994;331(26):1735-1738.

5. Merrer J, De Jonghe B, Golliot F, et al. Complications of femoral and subclavian venous catheterization in critically ill patients: A randomized controlled trial. JAMA. 2001;286(6):700-707.

6. Steele R, Irvin CB. Central line mechanical complication rate in emergency medicine patients. Acad Emerg Med. 2001;8(2):204-207.

7. Calvache JA, Rodriguez MV, Trochez A, Klimek M, Stolker RJ, Lesaffre E. Incidence of mechanical complications of central venous catheterization using landmark technique: Do not try more than 3 times. J Intensive Care Med. 2016;31(6):397-402.

8. Barsuk JH, McDaghie WC, Cohen ER, Balachandran JS, Wayne DB. Use of simulation-based mastery learning to improve the quality of central venous catheter placement in a medical intensive care unit. J Hosp Med. 2009;4(7):397-403.

9. American Society of Anesthesiologists Task Force on Central Venous Access, Rupp SM, Apfelbaum JL, et al. Practice guidelines for central venous access: A report by the American Society of Anesthesiologists Task Force on Central Venous Access. Anesthesiology. 2012;116(3):539-573.

10. Bodenham Chair A, Babu S, Bennett J, et al. Association of Anaesthetists of Great Britian and Irealand: Safe vascular access 2016. Anaesthesia. 2016;71:573-585.

11. Frykholm P, Pikwer A, Hammarskjöld F, et al. Clinical guidelines on central venous catheterisation. Swedish Society of Anaesthesiology and Intensic Care Medicine. Acta Anaesteshiol Scand. 2014;58(5):508-524.

12. Sekiguchi H, Tokita JE, Minami T, Eisen LA, Mayo PH, Narasimhan M. A prerotational, simulation-based workshop improves the safety of central venous catheter insertion: Results of a successful internal medicine house staff training program. Chest. 2011;140(3): 652-658

13. Dong Y, Suri HS, Cook DA, et al. Simulation-based objective assessment discerns clinical proficiency in central line placement: A construct validation. Chest. 2010;137(5):1050-1056.

14. Evans LV, Dodge KL, Shah TD, et al. Simulation training in central venous catheter insertion: Improved performance in clinical practice. Acad Med. 2010;85(9):1462-1469.

15. Lefrant JY, Muller L, De La Coussaye JE et al. Risk factors of failure and immediate complication of subclavian vein catheterization in critically ill patients. Intensive Care Med. 2002;28(8):1036-1041.

16. Dariushnia SR, Wallace MJ, Siddigi NH, et al. Quality improvement guidelines for central venous access. J Vasc Interv Radiol. 2010;21(7):976-981.

17. Barnes GD, Lucas E, Alexander GC, Goldberger ZD. National trends in ambulatory oral anticoagulant use. Am J Med. 2015;128(12):1300-1305.e2. 